\title{
Editorial: Peregrine Soliton and Breathers in Wave Physics: Achievements and Perspectives
}

\author{
Bertrand Kibler ${ }^{1 *}$, Amin Chabchoub ${ }^{2,3,4}$ and Heremba Bailung ${ }^{5}$ \\ ${ }^{1}$ Laboratoire Interdisciplinaire Carnot de Bourgogne (ICB), UMR 6303 CNRS-Université Bourgogne Franche-Comté, Dijon, \\ France, ${ }^{2}$ Hakubi Center for Advanced Research, Kyoto University, Kyoto, Japan, ${ }^{3}$ Disaster Prevention Research Institute, Kyoto \\ University, Kyoto, Japan, ${ }^{4}$ Centre for Wind, Waves and Water, School of Civil Engineering, The University of Sydney, Sydney, \\ NSW, Australia, ${ }^{5}$ Plasma Physics Laboratory, Physical Sciences Division, Institute of Advanced Study in Science and Technology, \\ Guwahati, India
}

Keywords: nonlinear waves, hydrodynamics, optics, plasma physics, breathers and rogue waves, nonlinear Schrodinger equation

Editorial on the Research Topic

Peregrine Soliton and Breathers in Wave Physics: Achievements and Perspectives

\section{OPEN ACCESS}

Edited and reviewed by: José S. Andrade Jr, Federal University of Ceara, Brazi

*Correspondence: Bertrand Kibler

bertrand.kibler@u-bourgogne.fr

Specialty section:

This article was submitted to Mathematical and Statistical Physics,

a section of the journal

Frontiers in Physics

Received: 15 October 2021 Accepted: 04 November 2021

Published: 02 December 2021

Citation:

Kibler B, Chabchoub A and Bailung $H$ (2021) Editorial: Peregrine Soliton and Breathers in Wave Physics: Achievements and Perspectives. Front. Phys. 9:795983. doi: 10.3389/fphy.2021.795983
The field of rogue wave physics has been extended well beyond the hydrodynamics community during the last decade, in particular, due to the analogy drawn through the one-dimensional nonlinear Schrödinger equation (NLSE) and related nonlinear wave theory. More specifically, breather solutions to the NLSE, whose entire space-time evolution is analytically described, are now considered as the simplest nonlinear prototypes of possible eminent hydrodynamic rogue waves, which are responsible for many maritime catastrophes. A special focus has been placed on doubly localized Peregrine-type breathers. Breathers exhibit unique pulsating on a nonzero background and localization properties directly linked to the ubiquitous modulation instability phenomenon in one-dimensional wave propagation scenarios.

It is worth mentioning that since their discovery in the late $70 \mathrm{~s}$, breather solutions on a finite background and notably those manifesting double localization [1] have remained untested experimentally, for almost 30 years, until their emergence into the light in 2010 [2]. Following the observation in optics, two experimental observations in other distinct fields of wave physics, namely, fluids dynamics [3] and plasma [4], followed within a few months after. All of them confirmed the controlled generation of doubly localized and strongly nonlinear Peregrine-type waves in real physical systems. These have now been among the highly cited studies in nonlinear wave physics during the last decade. The proof of existence and control of this class of nonlinear waves have been of fundamental significance for extreme wave dynamics control in laboratory environments and are now driving numerous experimental studies in various wave systems and mathematical and engineering developments worldwide.

This multidisciplinary research topic commemorates the 10th anniversary of the observation of the Peregrine breather (also commonly called the Peregrine soliton or the rational breather). A collection of fourteen original research, four brief research report, three mini-review, and three review articles are presented, featuring some of the latest advances in theoretical, numerical, and experimental studies of breather waves and modulation instability processes.

As an introduction to this research topic, we first refer the reader to four articles that review some of the theoretical foundations of breather waves. Karjanto discussed the relationship between periodic first-order breather solutions and their limiting behavior toward the Peregrine breather. Alejo et al. recalled the unstable properties of these nonlinear Schrödinger-type breathers, according 
to a standard definition of stability. The work by Akhmediev focused on the Peregrine breather as an "elementary particle" of more complicated patterns resulting from its higher-order combination. Such solutions are characterized by "triangular numbers," defined as the total number of Peregrine waves in a regular pattern, while Osborne elaborated on how to determine the general breather solutions by means of Riemann theta functions.

Next, more recent and specific theoretical findings can be found in the following seven articles addressing complex wave propagation problems. The study by Pelinovsky provided a methodology to compute the instability rates for the standing periodic waves and the double-periodic solutions of the NLSE. Ye et al. presented exact explicit coupled Peregrine solutions on a periodic-wave background caused by the interference in the vector cubic-quintic nonlinear Schrödinger equation. Uthayakumar et al. reviewed the Peregrinetype solutions appearing under the framework of extended versions of the NLSE describing the diverse nonlinear systems. In addition, Peng et al. looked into the dynamics of pole trajectories in the complex plane for rational solutions of the NLSE and its extensions. El-Tantawy et al. investigated breather waves in a linear damped nonlinear Schrödinger equation. Schober and Islas explored the effects of dissipation and higher-order nonlinearities on the stability of breather solutions, whilst Singh and Saini examined modulation instability and breathers in a dusty plasma.

In parallel, the reader can find four articles with detailed numerical investigations of both fundamental and applied problems, for which no analytical development is yet available. The contribution of Agafontsev and Gelash analyzed the spontaneous emergence of rogue waves for two different initial wave systems, namely, the unstable plane wave and the bound-state multi-soliton. Coulibaly et al. reported on the possible persistence of Peregrine-type waves in systems outside of the equilibrium, as described by the driven and damped nonlinear Schrödinger equation. From a more applied perspective, Shou and Huang proposed a scheme to actively control optical Peregrine breathers in a coherent atomic gas via electromagnetically induced transparency, while Wang et al. investigated the probability of extreme waves in deep crossing random seas in response to the variation of initial spectral bandwidth.

Furthermore, the reader will be able to discover eight articles providing recent experimental observations and characterizations of breather or rogue wave dynamics using distinct laboratory facilities to generate water, ion-acoustic, and light waves. Chabchoub et al. showed the possible observation of the degenerate soliton interaction, also demonstrated to be a Peregrine breather on the zero-background limit. The brief report by $\mathrm{Hsu}$ et al. demonstrated that the Peregrine breather traveling at the free surface of a shear current of slowly varying vorticity may transform into gray solitons. Using a multidipole double plasma device, Pathak analyzed the effect of Landau damping on the evolution of the ion-acoustic Peregrine breather. Naveau et al. described a novel optical fiber experimental setup based on a heterodyne optical time-domain reflectometer. Here, breather and recurrence dynamics can be evidenced by monitoring the power and relative phase evolutions of spectral components. Tikan et al. reviewed optical fiber experiments involving either single pulse or partially coherent waves that show the spontaneous and local emergence of the Peregrine breather. $\mathrm{Xu}$ et al. reported the observation of a novel type of breather interaction in telecommunication optical fibers, namely, ghost-like breather interaction dynamics, in which two identical breathers propagate with opposite group velocities. The work of Finot stressed and illustrated that great care must be taken when trying to identify the nature of coherent structures in an experimental record. An example of a highly peaked structure over a continuous background is shown to have any connection with a Peregrine breather, despite possessing similar key features. Jauberteau et al. showed the possible boosting or taming of spatiotemporal beam instabilities and the possible formation of rogue optical filaments in quadractic crystals.

Last but not least, it is worth to highlight the original research of Waseda et al. in a real sea environment, making use of a stereo image sequence of the ocean surface. With the help of a nonlinear phase resolving wave model, they evidenced a coherent wave group, compact in both propagating and transverse directions and oriented obliquely to the propagation direction, similarly to the recently discovered directional solitons and breathers.

In conclusion, this research topic contains 24 articles devoted to the multifaceted development of ongoing studies in the field of nonlinear Schrödinger breathers and related modulation instability. We strongly hope that this "research topic" will serve as a useful collection of state-of-the-art accomplishments of the research community to date. As such, we anticipate that these contributions will lead to a growing interest, research inspiration, and significant advances, which will be benefiting a wide range of theoretical and applied scientists.

\section{AUTHOR CONTRIBUTIONS}

All authors listed have made a substantial, direct, and intellectual contribution to the work and approved it for publication.

\section{FUNDING}

This work has received funding from the French National Research Agency (PIA2/ISITE-BFC, Grant No. ANR-15-IDEX03, "Breathing Light" project).

\section{ACKNOWLEDGMENTS}

The Research Topic editors thank all of our contributing authors for their dedication to the Research Topic and the reviewers for their constructive comments and suggestions. 


\section{REFERENCES}

1. Peregrine DH. Water Waves, Nonlinear Schrödinger Equations and Their Solutions. J Aust Math Soc Ser B, Appl. Math (1983) 25(1):16-43. doi:10.1017/s0334270000003891

2. Kibler B, Fatome J, Finot C, Millot G, Dias F, Genty G, et al. The Peregrine Soliton in Nonlinear Fibre Optics. Nat Phys (2010) 6(10):790-5. doi:10.1038/ nphys 1740

3. Amin C, Hoffmann NP, Akhmediev N. Rogue Wave Observation in a Water Wave Tank. Phys Rev Lett (2011) 106(20):204502.

4. Bailung H, Sharma SK, Nakamura Y. Observation of Peregrine Solitons in a Multicomponent Plasma with Negative Ions. Phys Rev Lett (2011) 107(25):255005. doi:10.1103/ physrevlett.107.255005
Conflict of Interest: The authors declare that the research was conducted in the absence of any commercial or financial relationships that could be construed as a potential conflict of interest.

Publisher's Note: All claims expressed in this article are solely those of the authors and do not necessarily represent those of their affiliated organizations, or those of the publisher, the editors, and the reviewers. Any product that may be evaluated in this article, or claim that may be made by its manufacturer, is not guaranteed or endorsed by the publisher.

Copyright (c) 2021 Kibler, Chabchoub and Bailung. This is an open-access article distributed under the terms of the Creative Commons Attribution License (CC BY). The use, distribution or reproduction in other forums is permitted, provided the original author(s) and the copyright owner(s) are credited and that the original publication in this journal is cited, in accordance with accepted academic practice. No use, distribution or reproduction is permitted which does not comply with these terms. 